\title{
Wetting and spreading of droplets on rough aluminum surfaces
}

\author{
Anastasia Islamova*, and Konstantin Ponomarev \\ National Research Tomsk Polytechnic University, 634050 Tomsk, Russia
}

\begin{abstract}
The wetting and spreading of distilled water droplets on abrasion-treated aluminum alloy AlMg 6 surfaces were studied. Using the shadow method, the dependences of the dynamic contact angle and hysteresis on the arithmetic mean of the profile deviation were obtained. Based on the analysis of the height and hybrid 3D roughness parameters, a relationship between the texture and spreading of the droplet is established.
\end{abstract}

\section{Introduction}

Wetting and spreading is central to many natural phenomena and industrial operations such as coating, spray cooling, lubrication etc. [1-4]. Considerable amount of paper is devoted to study liquid spreading along the smooth solid surface experimentally [5-7], numerically [8,9] and analytically [10]. However, the surface of a solid is not perfectly smooth, but covered with irregularities of various shapes (pores, microcracks, local deformations, etc.). One of the most important scientific direction is to study wetting of engineering metal surfaces used in different industrial applications and liquid spreading over them.

The influence of a relief of engineering rough surfaces (aluminium alloy, iron alloy, copper, ceramic, plastic (poly-methylmethacrylate: PMMA) and titanium alloy) on wetting were studied based on the two-dimensional roughness parameters [11, 12]. The lastmentioned characterize only chosen profile and do not allow to evaluate the change in the relief along the surface in two coordinate directions. Another phenomenon influenced by the surface roughness is the contact angle hysteresis (CAH) [13], which is the difference between the advancing and receding dynamic contact angles (DCA). The CAH is possible to use for estimating the state of a solid surface. The surface roughness and CAH were found to effect significantly on the spreading of droplets with characteristic sizes of several millimeters $[14,15]$. There are studies of the surface wetting and influence of the CAH in different gravity levels [16]. Thus, it is important to study spreading of a liquid droplet over rough surfaces taking into account the value of the $\mathrm{CAH}$, which can vary depending on the roughness and irregularity locations.

The aim of the presented work is to establish connections between the texture of the aluminum surface formed by abrasive processing and its wetting and spreading of the water droplet.

\footnotetext{
${ }^{*}$ Corresponding author: agi2@tpu.ru
} 


\section{Methods and materials}

Experimental studies of spreading and wetting have been conducted using the shadow method $[17,18]$. The volume $(10 \mu \mathrm{l})$ and flow rate $(5 \mu \mathrm{l} / \mathrm{s})$ of distiled water droplet were controlled.

Six AlMg6 aluminum alloy samples were used in the experiment. The samples are disks (50 $\mathrm{mm}$ in diameter and $4 \mathrm{~mm}$ thick). The substrates were polished using diamond pastes and abrasive felt wheels. Five samples were treated by abrasive discs (P2500, P1000, P800, P600, P400), after polishing. Surface roughness parameters are determined on the obtained 3D surface images (Fig. 1) using the profilometric complex "Micro Measure 3D station".

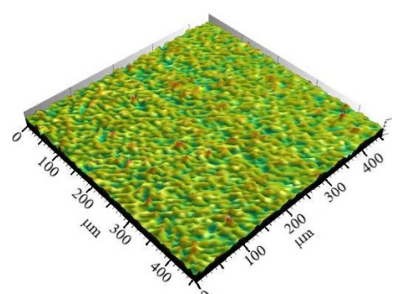

(a)

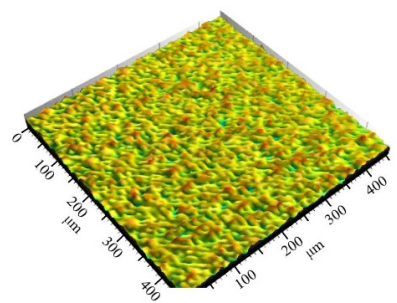

(d)

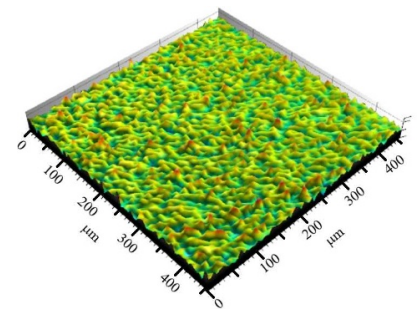

(b)

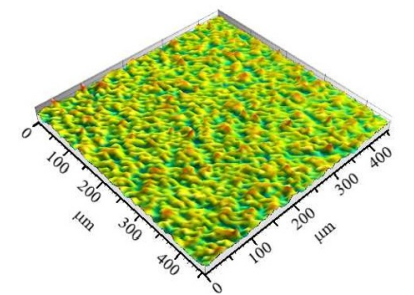

(e)

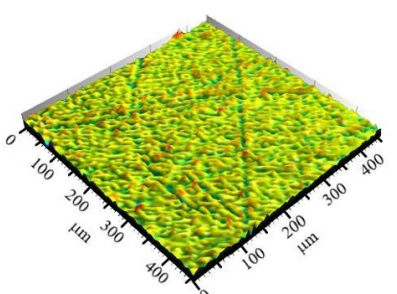

(c)

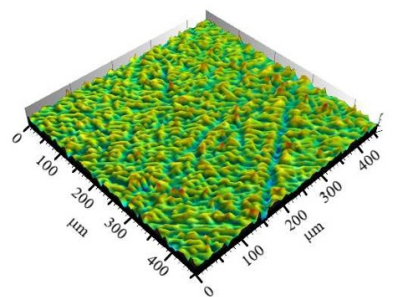

(f)

Fig. 1. 3D images of substrates. Untreated by abrasive discs: (a) Sample No 1 - polished. Treated by abrasive discs: (b) Sample No 2 - P2500, (c) Sample No 3 - P1000, (d) Sample No 4 - P800, (e) Sample No 5 - P600, (f) Sample No 6-P400.

The profilometer works as follows. A light beam with a diameter of $2 \mu \mathrm{m}$ illuminates the surface with a frequency of $30 \mathrm{~Hz}, 100 \mathrm{~Hz}, 300 \mathrm{~Hz}$ or $1000 \mathrm{~Hz}$. The frequency of light pulses is selected depending on the intensity of the reflected light and the surface condition (roughness, degree of gloss or blackness). A halogen lamp generates a beam of light. Then, along the fiber-optic conductor, the beam passes into the sensor, where it is formed into a ray. The height of the protrusions and depressions is determined from the difference between the intensities of the incident and reflected light. The surface roughness is calculated from the profile recorded on a segment of length. A three-dimensional image of the surface is formed as a result of repeated, sequential sample surface movement under the sensor. The analysis of the received profiles is performed using a computer program. It is established that after the abrasive treatment a texture (Samples No 2-6) is formed by chaotically located depressions and protrusions.

\section{Results and discussion}

Three-dimensional roughness characteristics were analyzed (Fig. 2). 3D surface parameters are chosen to describe the surface texture. In contrast to the two-dimensional parameters ( $R a, R z$, etc.), they characterize by not the selected profile, but the surface. The following parameters are selected: height (Ssk - skewness, $S k u$ - kurtosis, $S a$ - arithmetic mean 
height, $S q$ - root mean square (RMS) height) and hybrid ( $S d q$ - gradient of RMS, $S d r$ developed interracial area ratio).
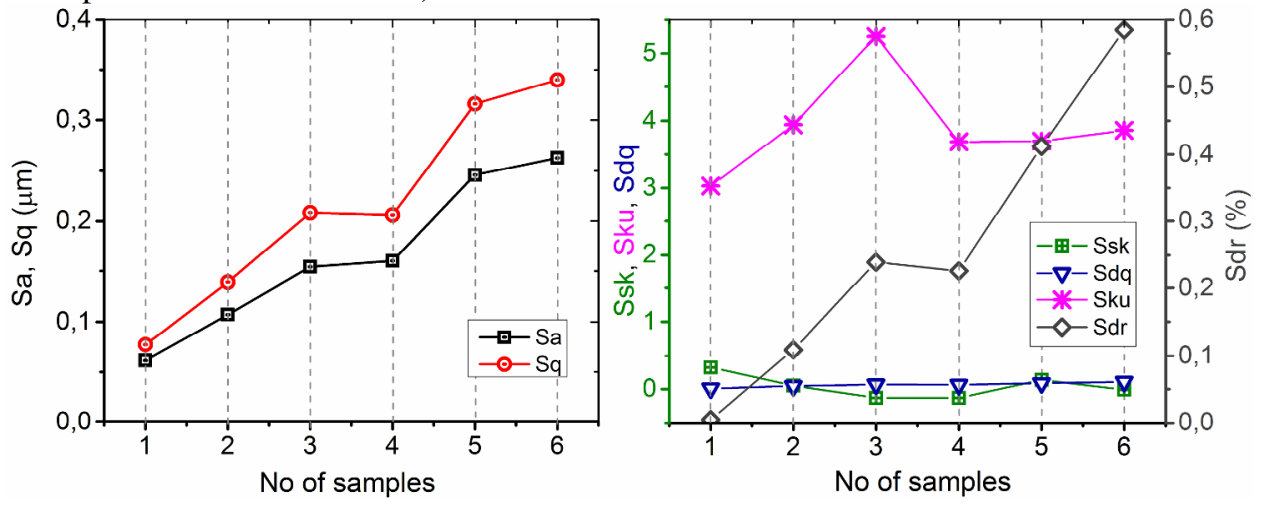

Fig. 2. Roughness parameters.

The parameters $S a$ and $S q$ are an indication of the general amplitude properties of the surface. It does not allowing to distinguish between pits and peaks. However it is convenient to compare surfaces obtained by one processing method. According to the obtained results, the polished surface is characterized by $S q$ and $S a$ parameters whose values are less than on abrasive-treated surfaces. It has also been established that after processing aluminum surfaces by abrasive disks with a larger grain, the parameters $S q$ and $\mathrm{Sa}$ become larger.

The skewness $S s k$ and kurtosis $S k u$ characterize the heights symmetry of the irregularities with respect to the median plane. Samples No 1 and 6 are characterize by $S s k=-0.0221$ and -0.00823 , respectively. It means that the distribution of unevenness heights of the nanometer scale is close to the symmetrical form. For samples No 3, 4 $S s k=-0.13$, the surface is characterized by pits. Positive values of the parameter Ssk (samples No 2 and 5) illustrate that high irregularities are arranged on the surface relative to shallow depressions.

The polished sample is characterized by $S k u=3.03$, irregularities have a symmetrical distribution. For samples 2-6, the values of $S k u$ are more than 3. The last-mentioned shows that the tops of the borders are higher than the Gaussian distribution (the peaked texture).

The relative change of surface area in its studied area relative to the nominal area $S d r$. $S d r=0.009 \%$ corresponds to the polished sample. For samples 2-6, the value of $S d r$ grows, since the spatial complexity of the texture increases.

Gradient of RMS $S d q$ is used mainly to assess the quality of wearing surface, surfaces with special requirements for their wetting by various liquids, characteristics analysis of reflecting surface and appearance (shine). The parameter $S d q$ can be considered a measure of surface smoothness, which agrees with the results: for the smoothest (polished) $S d q=0.009$, for the roughest one is 0.109 .

The effect of roughness on the surfaces wetting properties is analyzed. Fig. 3 shows the dependences of the static contact angle, the advancing and receding DCA, the hysteresis of the contact angle on the arithmetic mean height. 


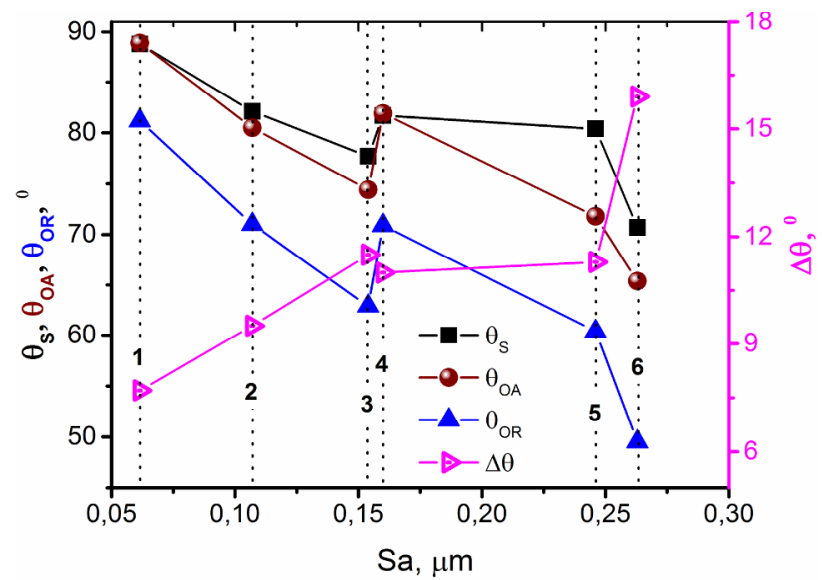

Fig. 3. Dependences of static contact angle $\theta_{\mathrm{s}}$, advancing $\theta_{\mathrm{OA}}$ and receding $\theta_{\mathrm{OR}} \mathrm{DCA}$, hysteresis of the contact angle $\Delta \theta$ on the arithmetic mean height.

It is seen from Fig. 3 that with growth in the surface roughness estimated by the parameter $S a$, the static contact angle decreases (agrees with the Wenzel-Derjaguin equation $\left(\cos \theta=r \cos \theta_{0}\right)$ [19]). According to this relation, if the liquid wets the material, an increase in the roughness coefficient causes a decrease in the macro-contact angle. However, for surfaces 3 and 4 , the parameters $S a$ and the static angles differ insignificantly, which lies within the confidence interval.

Based on the results of the conducted studies of the spreading and flow-out process, hysteresis of the contact angle, the advancing and receding DCA are determined. The pinning of contact line is registered, it follows that the hysteresis of the contact angle on all surfaces is not zero. The roughness leads to the appearance of metastable equilibrium of the system. Macro-contact angles on a rough surface depend on the direction of the liquid flow, since the position of the wetting line in a state of metastable equilibrium is different for advancing and receding. Therefore, the roughness is one of the main causes of wetting hysteresis. It is found that the contact angle hysteresis grows with increasing $S a$.

For samples 2-6, the static contact angles do not lie in the interval of advancing and receding DCA ( $\theta_{\mathrm{OR}} \leq \theta \leq \theta_{\mathrm{OA}}$ ), except polished. According to the molecular-kinetic theory [20], when a droplet is placed on surface, it reaches an equilibrium state when all of its kinetic energy is dissipated. If the hard surface is rough enough, i.e. the frictional forces in the displacement of the contact line are large, and then the entire kinetic energy of the drop dissipates to a thermodynamic equilibrium state. Therefore, pinning of the contact line on the irregularities will occur, which will lead to a larger contact angle than expected. Most likely, this explains why the obtained static angles are greater than the limiting upcoming DCA and do not fall within the ranges $\theta_{\mathrm{OR}} \leq \theta \leq \theta_{\mathrm{OA}}$ for all rough surfaces.

\section{Conclusion}

Three-dimensional roughness characteristics were analyzed. 3D surface parameters are chosen to describe the surface texture. Dependences of static, dynamic contact angles and hysteresis are obtained when a drop of distilled water spreads on abrasive-treated aluminum surfaces. With an increase in the parameter $\mathrm{Sa}$, the static contact angle decreases, which agrees with the Wenzel-Deryagin equation [19]. The contact angle hysteresis increases with the growth of surface roughness (arithmetical mean height $\mathrm{Sa}$ ). Static contact angles do not 
lie in the range of advancing and receding DCAs, i.e. the expression $\theta_{\mathrm{OR}} \leq \theta \leq \theta_{\mathrm{OA}}$ is not valid for all surfaces except polished.

The reported study was supported by RFBR, research project No. 18-38-00315 mol_a.

\section{References}

1. A. Sivkov, Y. Shanenkova, A. Saigash, I. Shanenkov, Surf. Coat. Technol. 292, 63 (2016)

2. A.V. Dmitriev, O.S. Dmitrieva, I.N. Madyshev, Therm. Eng. 63(9), 674 (2016)

3. V.E. Nakoryakov, S.Y. Misyura, J Eng Thermophys. 25(1), 24 (2016)

4. O.V. Vysokomornaya, M.V. Piskunov, P.A. Strizhak, Appl. Therm. Eng. 127, 1340 (2017)

5. G.V. Kuznetsov, D.V. Feoktistov, E.G. Orlova, S.Y. Misyura, V.S Morozov, A.G. Islamova, Int J Heat Mass Transf. 126, 161 (2018)

6. R.S. Volkov, P.A. Strizhak, S.Y. Misyura, S.I. Lezhnin, V.S. Morozov, Exp. Therm Fluid Sci. 99, 59 (2018)

7. Y.H. Kim, K. Kim, J.H. Jeong, Int J Heat Mass Transf. 102, 826 (2016)

8. X. Shang, Z. Luo, E.Y. Gatapova, O.A. Kabov, B. Bai, Comput. Fluids, 172, 181 (2018)

9. K.A. Raman, R.K. Jaiman, Th.-S. Lee, H.-T. Low, Chem. Eng. Technol. 145, 181 (2016)

10. J. Long, M.N. Hyder, R.Y.M. Huang, P. Chen, Adv. Colloid Interface Sci. 118, 173 (2005)

11. K. J. Kubiak, M. C. T. Wilson, T. G. Mathia, S. Carras, Scanning, 33, 370 (2011)

12. K.J. Kubiak, M.C.T. Wilson, T.G. Mathia, Ph. Carval, Wear, 271, 523 (2011)

13. E.B. Dussan V. Annu, Rev. Fluid Mech. 11, 371 (1979)

14. D. Sivakumar, K. Katagiri, T. Sato, H. Nishiyama, Phys. Fluids, 17, 100608 (2005)

15. H. Song, Y. Lee, S. Jin, H.-Y. Kim, J.Y. Yoo, Microelectron. Eng. 88(11), 3249 (2011)

16. O. Kabov, D. Zaitsev, Doklady Physics, 58(7), 292 (2013)

17. E.G. Orlova, D.V. Feoktistov, G.V. Kuznetsov, K.O. Ponomarev Eur. J. Mech. B/Fluids 68, 118 (2018)

18. I. Afanasyev, E.G. Orlova, D.V. Feoktistov, EPJ Web Conf. 82, 01054 (2015)

19. B.D. Summ, Yu.V. Goryunov, Physical and Chemical Basics of Wetting and Spreading (Khimiya, Moscow, 1976) [in Russian]

20. R. Fetzer, J. Ralston, J. Phys. Chem. C 113, 8888 (2009) 